\title{
OPTIMAL CONTROL APPROACH IN INVERSE RADIATIVE TRANSFER PROBLEMS: THE PROBLEM ON BOUNDARY FUNCTION ${ }^{*}, * *$
}

\author{
Valeri I. Agoshkov ${ }^{1}$ And Claude Bardos ${ }^{2}$
}

\begin{abstract}
The paper presents some results related to the optimal control approachs applying to inverse radiative transfer problems, to the theory of reflection operators, to the solvability of the inverse problems on boundary function and to algorithms for solution of these problems.
\end{abstract}

AMS Subject Classification. 49, 35Q.

Received November 13, 1998. Revised December 22, 1999 and March 17, 2000.

\section{INTRODUCTION}

Mathematical theory of radiative transfer problems and kinetic equations is nowadays an extensive area of mathematical physics $[1,2,5,8-10,15]$. It has various applications in astrophysics, theory of nuclear reactors, geophysics, theory of chemical processes, semiconductor theory, etc. Radiative field in these problems is defined by functions $\phi$ of spectral intensities of radiations which vary in accordance with transport equations. One-velocity (monenergetic) stationary transport equations constitute an important case of partial integrodifferential equations that arise in th eproblems of neutron spread, in the transfer of optical radiation and in other fields of physics $[1,2,9,10]$.

Let stationary radiative transfer be considered in a convex three-dimensional domain $D$ with the boundary $\partial D$; the unit vector $s=\left(s_{1}, s_{2}, s_{3}\right)$ is oriented in the direction of radiative transfer and it is determinated by polar angle $\vartheta \in[0, \pi]$ and azimuth $\varphi \in[0,2 \pi] ; \Omega$ is the unit sphere; $n$ is an unit vector of outher normal to $\partial D$. We consider a stationary transport equation in the following form:

$$
A \phi=f \quad \text { in } \Omega \times D,
$$

where $f$ is an internal source function and $A$ is the transport equation operator. Let $D$ be situated in a medium from which an "incoming flux" $\phi_{(\Gamma)}$ falls on $D$. Then the following boundary condition to $\phi$

$$
\phi=\phi_{(\Gamma)} \quad \text { on } \quad \partial D \quad \text { as } \quad s \cdot n<0
$$

\footnotetext{
Keywords and phrases: Optimal control, inverse problem, inverse radiative transfer problem, reflection operator, control equation operator, regularization parameter, iterative algorithm.

* This work was supported by the Russian Foundation for the Basic Research (Grant 97-01-00275).

** Ministère de l'Éducation Nationale (France) and CNRS while visiting the Émile Borel Center.

${ }^{1}$ Institute of Numerical Mathematics, Russian Academy of Sciences, Moscow, Russia, and CMLA, ENS Cachan, France; e-mail: agoshkov@inm.ras.ru

2 CMLA, ENS Cachan, France.
} 
can be imposed. The boundary-value problem $(1.1,1,2)$ is "the direct problem". It may be well- or ill-posed. Direct radiative problems are usually well-posed $[5,8,26]$.

We assume $\phi_{(\Gamma)}$ to be unknown while an "outgoing flux" $\phi \equiv \phi_{\text {obs }}$ is observed on $\partial D$ as $s \cdot n>0$. Let us consider one of inverse radiative problems: given $f, \phi_{\text {obs }}$ find $\phi$ and $\phi_{(\Gamma)} \equiv v$ such that

$$
\begin{array}{rlrl}
A \phi & =f & \text { in } \quad \Omega \times D, \\
\phi & =v \quad \text { on } \partial D \text { as } s \cdot n<0 \\
\phi & =\phi_{\text {obs }} & \text { on } \partial D \text { as } & s \cdot n>0 .
\end{array}
$$

A number of physical problems can be formulated in the form (1.3) or can be approximately reduced to it: the problem on "a critical incoming flux" such that $D$ radiates an outgoing flux $\phi=\phi_{\text {obs }}$ which is "limiting"; the problem on radiation in the half-space domain with the following additional condition - the plane at $z=0$ radiates a given flux $\phi=\phi_{\text {obs }}$ which is determinated by a given temperature $T_{\text {obs }}$ on this plane, and other problems.

The boundary-value problem (1.3) is the particular case of a wide class of inverse radiative transfer problems $[1,2,10,15,17,18,20,21,24]$. Usually inverse problems are ill-posed and there are specific difficulties in development and numerical solution of such problems. They are often considered under additional assumptions on $D$, coefficients, source functions, etc. [15-18, 21-24]. Therefore the elaboration of new approaches in this field is an actual problem. One of these approaches may be based on the optimal control theory [3]. Indeed, the problem (1.3) can be considered as "an exact controllability problem" to the state-equation solution $\phi$ and to the "control" $v$. Hence, to investigate (1.3) general results of the control theory and operator equations (of first- or second-type) can be used. Unfortunately, a lot of fine controllability results for elliptic, parabolic and hyperbolic equations can not be used here and one needs new "mathematical tools" to develop inverse radiative transfer problems by optimal control theory approaches. In this paper we consider one of such approaches and corresponding "tools" applying simple inverse problem on unknown boundary function of type (1.3).

We show also the significance of "reflection operators" $[6,11]$ for development of inverse radiative problems. These operators can be considered as analogous of Poincaré-Steklov operators in the radiative transfer theory.

Hystorically, functions of special type called reflection and transmission functions were introduced and investigated in astrophysics [1,2]. More common conception, the reflection operators, was developed in [4$6]$.

So, the paper deals with the results related to a methodology of analysis of inverse radiative transfer problems and corresponding reflection operators, estimates for the norms of these operators and an iterative algorithm to solve the inverse problems on boundary function. This methodology includes the following main stages: statement of an inverse problem; reformulation it as an optimal control theory problem, where some of unknown functions are taken as "controls"; investigations of reflection operator properties and of the control equation operator; solvability results (particularly in the case as regularization parameters are equal to zero); numerical algorithms and their convergence rate.

We discuss this methodology applying to one of inverse radiative problems. But it can be applied to other inverse problems of mathematical physics [14, 28-31].

\section{Statement of the Problem}

2.1. Let the domain $D$ be bounded by two parallel planes which are perpendicular to the axis $O z$, i.e. $D$ is "the slab" of thickness $H$ and we will write $D=(0, H)$. Assume $f, \phi_{(\Gamma)}, \phi_{\mathrm{obs}}$ and all coefficients of one-velocity stationary transport equation to be depended on $z, \vartheta, \varphi$. Then the solution $\phi$ will be a function of these variables 
only and a class of radiative transfer problems in the slab $D=(0, H)$ can be reduced to the boundary-value problem $(1.1,1.2)$ which can be written as follows $[1,2]$ :

$$
\begin{gathered}
A \phi \equiv \mu \frac{\partial \phi}{\partial z}+\phi-\frac{b}{4 \pi} \int_{0}^{2 \pi} \int_{-1}^{1} p\left(z, \mu_{0}\right) \phi\left(\varphi^{\prime}, \mu^{\prime}, z\right) d \mu^{\prime} d \varphi^{\prime}=f(\varphi, \mu, z), \\
0<z<H, \quad-1 \leq \mu \leq 1, \quad 0 \leq \varphi \leq 2 \pi \\
\phi(\varphi, \mu, 0)=\phi_{(\Gamma)}^{(1)}(\varphi, \mu) \quad \text { for } \quad 0<\mu \leq 1, \quad 0 \leq \varphi \leq 2 \pi, \\
\phi(\varphi, \mu, H)=\phi_{(\Gamma)}^{(2)}(\varphi, \mu) \quad \text { for } \quad-1<\mu<0, \quad 0 \leq \varphi \leq 2 \pi,
\end{gathered}
$$

where $0 \leq b(z) \leq b_{1}=$ const $<1, H<\infty, \quad p\left(z, \mu_{0}\right)$ is the phase function characterizing the scattering in the direction $(\mu, \varphi)$ of radiation arriving from the direction $\left(\mu^{\prime}, \varphi^{\prime}\right)$ on the volume element at the point $z$, $\mu_{0}=\mu \mu^{\prime}+\left(1-\mu^{2}\right)^{1 / 2}\left(1-\mu^{\prime 2}\right)^{1 / 2} \cos \left(\varphi-\varphi^{\prime}\right),(\mu, \varphi) \in[-1,1] \times(0,2 \pi) \quad($ or $(\vartheta, \varphi) \in(0, \pi) \times(0,2 \pi)$, where $\vartheta=\arccos \mu$ ) is a direction of radiative transfering. We assume all functions considered below to be defined (with respect to variables $\vartheta, \varphi$ ) on the unit sphere $\Omega$ and to be periodical with respect to $2 \pi$-periodic argument $\varphi$. The points on $\Omega$ are given by the vector $s=\left(s_{1}, s_{2}, s_{3}\right)$ with components $s_{1}=\sin \vartheta \sin \varphi, s_{2}=\sin \vartheta \cos \varphi$, $s_{3}=\cos \vartheta$.

Below we consider the case when

$$
\begin{gathered}
\int_{0}^{2 \pi} \int_{-1}^{1}\left|p\left(z, \mu_{0}\right)\right| d \mu^{\prime} d \varphi^{\prime} \leq 4 \pi \forall z \in(0, H), p\left(z, \mu_{0}\right) \equiv p_{N}\left(z, \mu_{0}\right)=\sum_{l=0}^{N} w_{l}(z) P_{l}\left(\mu_{0}\right), \\
N<\infty, \quad w_{0}=1>w_{l} \geq 0,
\end{gathered}
$$

where $P_{l}(\mu) \equiv P_{l}^{(0)}(\mu), P_{l}^{(m)}(\mu)$ - Legendre polynomials (if $N=0$ then the isotropic scattering case is considered; $N=1$ corresponds to the " $P_{1}$-approximation" which is used systematically in the nuclear reactor theory, and so on). We will satisfy these constraints hereafter and use real-valued functions.

We introduce the following notations

$$
B_{0} \phi=\frac{1}{4 \pi} \int_{0}^{2 \pi} \int_{-1}^{1} p\left(z, \mu_{0}\right) \phi\left(\varphi^{\prime}, \mu^{\prime}, z\right) d \mu^{\prime} d \varphi^{\prime}, \quad L \phi=\mu \frac{\partial \phi}{\partial z}+\phi, \quad T \phi=\phi-b B_{0} \phi .
$$

If $\phi$ is a solution of $(2.1-2.3)$ then

$$
\phi(\mu, \varphi, z)=l^{-1} \phi_{(\Gamma)}+L^{-1} F \equiv \phi_{T}+L^{-1} F,
$$

where $\phi_{T}$ is a solution of the equation $l \phi_{T} \equiv L \phi_{T}=0$ with boundary conditions $(2.2,2.3)$ and

$$
\begin{gathered}
F(\mu, \varphi, z) \equiv b B_{0} \varphi+f, \\
\phi_{T} \equiv l^{-1} \phi_{(\Gamma)}=\left\{\begin{array}{l}
\phi_{(\Gamma)}^{(1)}(\mu, \varphi) e^{-\frac{z}{\mu}}, \mu>0, \\
\phi_{(\Gamma)}^{(2)}(\mu, \varphi) e^{\frac{(H-z)}{\mu}}, \mu<0,
\end{array}\right.
\end{gathered}
$$




$$
\begin{gathered}
L^{-1} F=\left\{\begin{array}{l}
\int_{0}^{z} e^{-\frac{z-z^{\prime}}{\mu}} F\left(\mu, \varphi, z^{\prime}\right) \frac{d z^{\prime}}{\mu}, \mu>0, \\
-\int_{z}^{H} e^{-\frac{\left(z^{\prime}-z\right)}{|\mu|}} F\left(\mu, \varphi, z^{\prime}\right) \frac{d z^{\prime}}{\mu}, \mu<0,
\end{array}\right. \\
\phi_{(\Gamma)} \equiv\left(\phi_{(\Gamma)}^{(1)}(\mu, \varphi), \phi_{(\Gamma)}^{(2)}(\mu, \varphi)\right) .
\end{gathered}
$$

Let us introduce the following sets:

$$
X=\{(\varphi, \mu, z): \varphi \in[0,2 \pi], \mu \in[-1,1], z \in(0, H)\},
$$

$\Gamma_{-}=\{(\varphi, \mu, z):(\varphi \in[0,2 \pi], \mu \in[0,1], z=0) \cup(\varphi \in[0,2 \pi], \mu \in[-1,0], z=H)\}$,

$\Gamma_{+}=\{(\varphi, \mu, z):(\varphi \in[0,2 \pi], \mu \in[-1,0], z=0) \cup(\varphi \in[0,2 \pi], \mu \in[0,1], z=H)\}$,

where $\Gamma_{-}$is the domain of inflow radiative transfer functions, while $\Gamma_{+}$is the domain of outflow radiative functions. Let $L_{2}, H_{2}^{1}$ be the functional spaces, where we define the inner products and norms as

$$
\begin{gathered}
(\phi, \psi)_{L_{2}} \equiv(\phi, \psi)=\int_{0}^{2 \pi} \int_{-1}^{1} \int_{0}^{H} u v d z d \mu d \varphi, \quad\|\phi\|_{L_{2}} \equiv\|\phi\|=(\phi, \phi)^{1 / 2} \\
(\phi, \psi)_{H_{2}^{1}}=(\phi, \psi)+\left(\mu \frac{\partial \phi}{\partial z}, \mu \frac{\partial \psi}{\partial z}\right), \quad\|\phi\|_{H_{2}^{1}}=\left(\|\phi\|^{2}+\left\|\mu \frac{\partial \phi}{\partial z}\right\|^{2}\right)^{1 / 2}
\end{gathered}
$$

where $\mu \partial \phi / \partial z \in L_{2}$ is a generalized derivative of $\phi \in L_{2}$. We define $L_{2,-} \equiv L_{2}\left(\Gamma_{-}\right)$as the space of vectorfunctions $\gamma_{(-)}(\varphi, \mu)=\left(\gamma_{(-)}^{(1)}(\varphi, \mu), \gamma_{(-)}^{(2)}(\varphi, \mu)\right)$ (the first components are defined for $\mu>0$ and the second ones for $\mu<0)$ with the norm

$$
\left\|\gamma_{(-)}\right\|_{L_{2,-}}=\left[\int_{0}^{2 \pi} \int_{0}^{1} \mu\left|\gamma_{(-)}^{(1)}(\varphi, \mu)\right|^{2} d \mu d \varphi+\int_{0}^{2 \pi} \int_{-1}^{0}|\mu|\left|\gamma_{(-)}^{(2)}(\varphi, \mu)\right|^{2} d \mu d \varphi\right]^{1 / 2}
$$

The space $L_{2,+} \equiv L_{2}\left(\Gamma_{+}\right)$is introduced as the space of of vector-functions $\gamma_{(+)}(\varphi, \mu)=\left(\gamma_{(+)}^{(1)}(\varphi, \mu), \gamma_{(+)}^{(2)}(\varphi, \mu)\right)$ (the first components are defined for $\mu<0$ and the second ones for $\mu>0$ ) with the norm

$$
\left\|\gamma_{(+)}\right\|_{L_{2,-}}=\left[\int_{0}^{2 \pi} \int_{-1}^{0}|\mu|\left|\gamma_{(+)}^{(1)}(\varphi, \mu)\right|^{2} d \mu d \varphi+\int_{0}^{2 \pi} \int_{0}^{1} \mu\left|\gamma_{(+)}^{(2)}(\varphi, \mu)\right|^{2} d \mu d \varphi\right]^{1 / 2}
$$

2.2. Let $\phi_{(\Gamma)}$ be the vector-function: $\phi_{(\Gamma)}=\left(\phi_{(\Gamma)}^{(1)}, \phi_{(\Gamma)}^{(2)}\right)$, whose components are present in boundary conditions (2.2) and (2.3).

Theorem $2.1[5,34]$. If $f \in L_{2}$ and $\phi_{(\Gamma)} \in L_{2,-}$, then: (1) There exists a unique function $\phi \in H_{2}^{1}$ which is the solution to problem (2.1-2.3). (2) The function $\phi$ satisfies equations (2.1) almost everywhere in $X$ condition (2.2) for almost all $\mu>0$, and condition (2.3) for almost all $\mu<0$. (3) For $\phi$, the following estimates hold: 


$$
C\left[\|f\|_{L_{2}}+\left\|\phi_{(\Gamma)}\right\|_{L_{2,-}}\right] \leq\|\phi\|_{H_{2}^{1}} \leq \tilde{C}\left[\|f\|_{L_{2}}+\left\|\phi_{(\Gamma)}\right\|_{L_{2,-}}\right], \quad C, \tilde{C}>0
$$

where $C$ and $\tilde{C}$ are independent of $\phi, f$, and $\phi_{(\Gamma)}$.

Theorem 2.2 [5]. If $f \equiv 0$ in (2.1-2.3), $\phi_{(\Gamma)} \in L_{2,-}$, then: (1) There exists a unique function $\phi \in H_{2}^{1}$ which is the solution to problem (2.1-2.3). (2) The function $\phi$ satisfies equation (2.1) almost everywhere in $X$ and boundary conditions (2.2) and (2.3) almost everywhere. (3) For $\phi$, the relationship

$$
\left(T^{-1} \mu \frac{\partial \phi}{\partial z}, \mu \frac{\partial \psi}{\partial z}\right)+(T \phi, \psi)+(\phi, \psi)_{L_{2,+}}=\left(\phi_{(\Gamma)}, \psi\right)_{L_{2,-}}
$$

is valid for an arbitrary function $\psi \in H_{2}^{1}$, and the following estimates hold:

$$
C\left\|\phi_{(\Gamma)}\right\|_{L_{2,-}} \leq\|\phi\|_{H_{2}^{1}} \leq \tilde{C}\left\|\phi_{(\Gamma)}\right\|_{L_{2,-}}, \quad C, \tilde{C}>0,
$$

where $C$ and $\tilde{C}$ are independent of $\phi$ and $\phi_{(\Gamma)}$.

Theorem 2.3 [5, 28]. The following estimate holds for the solution $\phi$ of the problem $(2.1-2.3)$ with $f \equiv 0$ :

$$
\frac{\|\phi\|_{L_{2,+}}^{2}}{\|\phi\|_{L_{2,-}}^{2}} \leq \frac{1-\sqrt{1-b_{1}^{2}}\left(\cosh \left(H \sqrt{1-b_{1}^{2}}\right)-1\right) / \sinh \left(H \sqrt{1-b_{1}^{2}}\right)}{1+\sqrt{1-b_{1}^{2}}\left(\cosh \left(H \sqrt{1-b_{1}^{2}}\right)-1\right) / \sinh \left(H \sqrt{1-b_{1}^{2}}\right)} .
$$

2.3. Assume the function $\gamma_{(-)} \equiv v-$ "a control function", to be unknown, while there is a given function $\phi_{\text {obs }}$ $\in L_{2,+}$ defined on $\Gamma_{+}$. Then we can formulate the following inverse problem: for given $f \in L_{2}(X), \phi_{\text {obs }} \in L_{2,+}$ find $u \in H_{2}^{1}, v \in L_{2,-}$ such that

$$
A u=f \text { in } X, u=v \text { on } \Gamma_{-}, \inf _{v} J_{1}(v, u),
$$

where

$$
J_{1}(v, u)=\alpha\|v\|_{L_{2,-}}^{2}+\left\|u-\phi_{\mathrm{obs}}\right\|_{L_{2,+}}^{2}, \alpha=\text { const } \geq 0 .
$$

Let $\phi^{(0)}$ be the solution of the problem given by

$$
A \phi^{(0)}=f \text { in } X, \quad \phi^{(0)}=0 \text { on } \Gamma_{-} .
$$

We express this solution in the following form:

$$
\phi^{(0)}=G_{0} f,
$$

where $G_{0}: L_{2}(X) \rightarrow H_{2}^{1}$ is the linear operator - the resolution operator of the problem (2.11). Now we can reformulate (2.8) as the following inverse problem to the function $\phi=u-\phi^{(0)}$ : for given $\phi^{(0)} \in H_{2}^{1}, \phi_{\text {obs }} \in L_{2,+}$ find $\phi \in H_{2}^{1}, v \in L_{2,-}$ such that

$$
A \phi=0 \text { in } X, \phi=v \text { on } \Gamma_{-}, \inf _{v} J(\phi, v),
$$

where

$$
J(\phi, v)=\alpha\|v\|_{L_{2,-}}^{2}+\left\|\phi-\left(\phi_{\mathrm{obs}}-\phi^{(0)}\right)\right\|_{L_{2,+}}^{2}, \alpha=\mathrm{const} \geq 0, \phi_{\mathrm{obs}} \in L_{2,+} .
$$

Later on we investigate the inverse problem (2.11). 


\section{REFLECTION OPERATORS AND THEIR PROPERTIES}

3.1. Introduce some operators to be used in the sequel.

Consider the following radiative transfer problem:

$$
A \phi=0 \text { in } X, \phi=v \text { on } \Gamma_{-},
$$

where $v \in L_{2,-}$ is a given function. This problem has a unique solution $\phi \in H_{2}^{1}$. We write $\phi$ in the following form:

$$
\phi=G_{1} v
$$

where $G_{1}: L_{2,-} \rightarrow H_{2}^{1}$ is a linear bounded operator.

Later on we use also the traces operators $P_{(-)}, P_{(+)}$:

$$
\begin{gathered}
\left.P_{(-)} \phi \equiv \phi\right|_{\Gamma_{-}}=(\phi(\mu, \varphi, 0), \phi(-\mu, \varphi, H)),\left.\quad P_{(+)} \phi \equiv \phi\right|_{\Gamma_{+}}=(\phi(-\mu, \varphi, 0), \phi(\mu, \varphi, H)) \\
\forall \phi \in H_{2}^{1}, \quad \mu>0, \quad \varphi \in(0,2 \pi) .
\end{gathered}
$$

According to the above statements the operators $P_{(-)}, P_{(+)}$are bounded.

Now, let us introduce the adjoint problem

$$
\begin{gathered}
A^{*} \psi \equiv-\mu \frac{\partial \psi}{\partial z}+\psi-\frac{b}{4 \pi} \int_{0}^{2 \pi} \int_{-1}^{1} p\left(z, \mu_{0}\right) \psi\left(\varphi^{\prime}, \mu^{\prime}, z\right) d \mu^{\prime} d \varphi^{\prime}=g, \\
\psi(\varphi, \mu, 0)=\psi_{(\Gamma)}^{(1)}(\varphi, \mu), \quad-1 \leq \mu<0,0 \leq \varphi \leq 2 \pi, \\
\psi(\varphi, \mu, H)=\psi_{(\Gamma)}^{(2)}(\varphi, \mu), \quad 0<\mu \leq 1,0 \leq \varphi \leq 2 \pi,
\end{gathered}
$$

where $\psi_{(\Gamma)}=\left(\psi_{(\Gamma)}^{(1)}, \psi_{(\Gamma)}^{(2)}\right) \in L_{2,+}$. This boundary value problem has a unique solution $\psi \in H_{2}^{1} \forall g \in L_{2}$, $\psi_{(\Gamma)} \in L_{2,+}$. We represent $\psi$ as $\psi=\tilde{G}_{0} g \forall g \in L_{2}$ for $\psi_{(\Gamma)} \equiv 0$, and $\psi=\tilde{G}_{1} \psi_{(\Gamma)} \forall \psi_{(\Gamma)} \in L_{2,+}$ for $g \equiv 0$, where the resolution operators $\tilde{G}_{0}: L_{2} \rightarrow H_{2}^{1}, \tilde{G}_{1}: L_{2,+} \rightarrow H_{2}^{1}$ are bounded.

By $P_{1}, P_{2}$ we denote the projection operators defined as follows: $P_{1} g=\left(g_{1}, 0\right), P_{2} g=\left(0, g_{2}\right) \quad \forall g=\left(g_{1}, g_{2}\right)$ $\in L_{2,-}\left(\right.$ or $\left.L_{2,+}\right)$. The subspace $L_{2,-}^{(0)} \equiv P_{1} L_{2,-} \subset L_{2,-}\left(\right.$ or $\left.L_{2,+}^{(0)} \equiv P_{1} L_{2,+} \subset L_{2,+}\right)$ consists of vectors $P_{1} g=\left(g_{1}, 0\right) \forall g \in L_{2,-}\left(\right.$ or $\left.\forall g \in L_{2,+}\right)$.

3.2. Let us consider the problem (3.1). This problem has a unique solution $\phi \in H_{2}^{1}$ and there is a trace $\left.\phi\right|_{\Gamma_{+}} \in L_{2,+}$. Assume $\left.w \equiv \phi\right|_{\Gamma_{+}}$to obtain the transmission-reflection operator

$$
S v=w, \quad S: L_{2,-} \rightarrow L_{2,+}
$$

that will be called for simplicity the reflection operator.

Let $U, S_{U}$ be the following operators

$$
U w=w(\varphi+\pi,-\mu), \quad w(\varphi, \mu) \in L_{2, \pm}, \quad S_{U}=U S
$$

Consider now the adjoint problem $(3.3-3.5)$ where $g \equiv 0$ and $\psi_{(\Gamma)}=\left(\psi_{(\Gamma)}^{(1)}, \psi_{(\Gamma)}^{(2)}\right) \in L_{2,+}$. This problem has a unique solution $\psi \in H_{2}^{1}$. Assuming $\left.v \equiv \psi\right|_{\Gamma_{-}} \in L_{2,-}$, we obtain the operators

$$
S^{*} \psi_{(\Gamma)}=v, \quad S^{*}: L_{2,+} \rightarrow L_{2,-}, \quad S_{(U)}^{*}=S^{*} U
$$


Theorem 3.1. The following relationships and properties hold:

(1) $S^{*}=(S)^{*}, \quad\left(S_{U}\right)^{*}=S_{(U)}^{*}$.

(2) The operator $S_{U}$ is symmetric in $L_{2,-}$ and $S_{(U)}^{*}$ in $L_{2,+}$.

(3) $S=U S^{*} U, \quad S^{*}=U S U$.

(4) The following estimate holds for the norm of the operator $S$ :

$$
\|S\|^{2} \leq(1-q) /(1+q) \equiv \gamma_{1}
$$

where

$$
q=\sqrt{\left(1-b_{1}^{2}\right)} \frac{\cosh \left(H \sqrt{1-b_{1}^{2}}\right)-1}{\sinh \left(H \sqrt{1-b_{1}^{2}}\right)} .
$$

Proof. Properties (1-3) can be easily obtained using the following relationships:

$$
0=(A \phi, \psi)=(\phi, \psi)_{L_{2,+}}-(\phi, \psi)_{L_{2,-}}+\left(\phi, A^{*} \psi\right)=(\phi, \psi)_{L_{2,+}}-(\phi, \psi)_{L_{2,-}},
$$

i.e.

$$
\left(\phi, \psi_{(\Gamma)}\right)_{L_{2,+}}=\left(\phi_{(\Gamma)}, \psi\right)_{L_{2,-},}, \quad\left(S \phi_{(\Gamma)}, \psi_{(\Gamma)}\right)_{L_{2,+}}=\left(\phi_{(\Gamma)}, S^{*} \psi_{(\Gamma)}\right)_{L_{2,-},},
$$

where $\phi$ and $\psi$ are solutions of problems (2.1-2.3) $(f=0)$ and (3.3-3.5), respectively. Estimate (3.8) is a simple corollary of Theorem 2.3.

3.3. In the general case operator $S$ is not compact. Indeed, suppose that $b(z) \equiv 0$, then

$$
\phi=G_{1} v=l^{-1} v \equiv \phi_{T}, S v \equiv S_{T} v=P_{(+)} \phi_{T}=g=\left(g_{1}, g_{2}\right) \in L_{2,+} \forall v \in L_{2,-}
$$

where $g_{1}(\varphi, \mu)=v_{2}(\varphi, \mu) e^{-\frac{H}{|\mu|}}, \mu<0$ and $g_{2}(\varphi, \mu)=v_{1}(\varphi, \mu) e^{-\frac{H}{|\mu|}}, \mu>0$. We see that $S \equiv S_{T}: L_{2,-} \rightarrow L_{2,+}$ is bounded, while $S_{T}$ is not compact.

Let us prove the compactness of "a part of $S$ ". Introduce this "part" as follows

$$
S_{R}=S-S_{T}
$$

To investigate the properties of $S_{R}$ let us write at first an expression for this operators.

From (2.5) we have:

$$
\begin{gathered}
\phi=\phi_{T}+\phi_{R}, \\
L \phi_{R}=b B_{0} \phi_{R}+b B_{0} \phi_{T}, \quad \phi_{R}=0 \text { on } \Gamma_{-}, \\
\phi_{R}=L^{-1} b B_{0} \phi_{R}+L^{-1} b B_{0} \phi_{T} \\
\phi_{R}=\sum_{i=0}^{\infty}\left(L^{-1} b B_{0}\right)^{i} L^{-1} b B_{0} \phi_{T}=\sum_{i=0}^{\infty}\left(L^{-1} b B_{0}\right)^{i} L^{-1} b B_{0} l^{-1} v=\left(I-L^{-1} b B_{0}\right)^{-1} L^{-1} b B_{0} \phi_{T} .
\end{gathered}
$$

So, $S_{R}=P_{(+)} \sum_{i=0}^{\infty}\left(L^{-1} b B_{0}\right)^{i} L^{-1} b B_{0} l^{-1}=P_{(+)}\left(L-b B_{0}\right)^{-1} b B_{0} l^{-1}$ and the following statement are valid.

Lemma 3.1. The operator $S$ can be represented as $S=S_{R}+S_{T}$, where

$$
S_{T}=P_{(+)} l^{-1}, S_{R}=P_{(+)} \sum_{i=0}^{\infty}\left(L^{-1} b B_{0}\right)^{i} L^{-1} b B_{0} l^{-1}=P_{(+)}\left(L-b B_{0}\right)^{-1} b B_{0} l^{-1} .
$$

Here, the "transmission part" $S_{T}$ of $S$ is a bounded operator from $L_{2,-}$ into $L_{2,+}$. The "reflection part" $S_{R}$ of $S$ is a compact operator from $L_{2,-}$ into $L_{2,+}$. 
Proof. The expressions for $S_{R}, S_{T}$ follows from the above considerations. One needs to prove the compactness of $S_{R}$ only. The operator $l^{-1}$ is bounded from $L_{2,-}$ into $H_{2}^{1}$, the operator $B_{0}$ is compact from $H_{2}^{1}$ into $L_{2}$ [5] and the operator $P_{(+)}\left(L-b B_{0}\right)^{-1} b$ is bounded from $L_{2}$ into $L_{2,+}$ (see, Th. 2.1 and trace theorems [5]). Hence, the operator $S_{R}: L_{2,-} \rightarrow L_{2,+}$ is compact.

3.4. Consider now a "specific case" of $S$ - the restriction of $S$ to the subspace $L_{2,-}^{(0)}$ that we denote by $S_{1}: S_{1} v \equiv S v \forall v \in L_{2,-}^{(0)}$. So,

$$
S_{1}=S P_{1}, S_{1}: L_{2,-}^{(0)} \rightarrow L_{2,+}, D\left(S_{1}\right)=L_{2,-}^{(0)}, R\left(S_{1}\right) \subset L_{2,+}
$$

It is easily seen that

where

$$
S_{1}=S_{11}+S_{21}
$$

$$
\begin{aligned}
& S_{11} \equiv P_{1} S_{R} P_{1}=P_{1} S P_{1}: L_{2,-}^{(0)} \rightarrow L_{2,+}^{(0)}, \\
& S_{21} \equiv P_{2} S_{R} P_{1}+P_{2} S_{T} P_{1}=P_{2} S P_{1}: L_{2,-}^{(0)} \rightarrow P_{2} L_{2,+} .
\end{aligned}
$$

Note, that $P_{1} S_{T} P_{1} v \equiv 0 \forall v \in L_{2,-}$. The estimates and statements proved above for $S$ are valid for $S_{1}$ also. Some estimates for $S_{21}$ are given by the following lemma:

Lemma 3.2 [28]. The estimates

$$
\left\|P_{2} S_{R} P_{1}\right\| \leq \frac{b_{1}}{\sqrt{1-b_{1}}} e^{-\frac{\left(1-b_{1}\right) H}{2}}, \quad\left\|S_{21}\right\| \leq \frac{b_{1}}{\sqrt{1-b_{1}}} e^{-\frac{\left(1-b_{1}\right) H}{2}}+e^{-H}
$$

are valid.

Consider the sequence $H=H_{j}, j=1,2, \ldots\left(H_{j} \rightarrow \infty\right)$. Let $\phi_{j}$ be the solution of $(3.1)$ as $H=H_{j}, v \in L_{2,-}^{(0)}$. Assume that $H_{i}>H_{j}$ for some $i, j$. We extend $\phi_{j}$ to $\left(X_{i} \equiv(0,2 \pi] \times[-1,1] \times\left(0, H_{i}\right)\right) \backslash X_{j}$ as follows

$$
\tilde{\phi}_{j}(\varphi, \mu, z)=\left\{\phi_{j}\left(\varphi, \mu, H_{j}\right) e^{-\left(z-H_{j}\right) / \mu}, \mu>0 ; 0, \mu<0\right\} .
$$

Then function $\varepsilon_{j i}=\tilde{\phi}_{j}-\phi_{i}$ is a solution of

$$
A \varepsilon_{j i}=f_{j} \text { in } X_{i}, \varepsilon_{j i}=0 \text { on } \Gamma_{-}^{i},
$$

where

$$
\begin{gathered}
f_{j}=\left\{0, z \in\left(0, H_{j}\right) ;-b B_{0} \tilde{\phi}_{j}, z \in\left(H_{j}, H_{i}\right)\right\} \\
\left\|f_{j}\right\|_{L_{2}\left(X_{i}\right)} \leq C b_{1} \cdot\left(\int_{0}^{2 \pi} d \varphi \int_{0}^{1} \mu\left|\phi_{j}\left(\varphi, \mu, H_{j}\right)\right|^{2} d \mu\right)^{1 / 2}=C b_{1}\left\|S_{21}^{(j)} v\right\|_{L_{2,+}} \\
\leq C b_{1}\left(e^{-H_{j}}+\frac{b_{1}}{\sqrt{1-b_{1}}} e^{-\frac{\left(1-b_{1}\right) H_{j}}{2}}\right)\|v\|_{L_{2,-}}^{(0)} \rightarrow 0 \text { as } H_{j} \rightarrow \infty
\end{gathered}
$$

and $S_{21}^{(j)} \equiv S_{21}$ as $H=H_{j}$. Then

$$
\left(\int_{0}^{2 \pi} \int_{-1}^{0}|\mu|\left|\varepsilon_{j i}\right|^{2} d \varphi d \mu\right)^{\frac{1}{2}}=\left\|\left(S_{11}^{(i)}-S_{11}^{j}\right) v\right\|_{L_{2,+}^{(0)}} \leq C\left\|\varepsilon_{j i}\right\|_{H_{2}^{1}\left(X_{i}\right)} \leq C\left\|f_{j}\right\|_{L_{2}\left(X_{i}\right)} \rightarrow 0, i, j \rightarrow \infty
$$


We introduce operator $S^{(\infty)}$ :

$$
S^{(\infty)} v \equiv S_{11}^{(\infty)} v=\lim _{j \rightarrow \infty} S_{11}^{(j)} v \forall v \in L_{2,-}^{(0)}, \quad S^{(\infty)}: L_{2,-}^{(0)} \rightarrow L_{2,+}^{(0)}
$$

Lemma 3.3. Operator $S^{(\infty)}$ is compact.

Proof. Since the operators $S_{11}^{(j)}, j=1,2, \ldots$, are compact then $S^{(\infty)}=\lim _{j \rightarrow \infty} S_{11}^{(j)}$ is compact also.

Remark. The compactness of $S^{(\infty)}$ is proved also in [33].

Lemma 3.4. Let $\phi=G_{1} v$ be a solution of (3.1) as $H=\infty$ and $v \in L_{2,-}^{(0)}$. Then

$$
\left\|S^{(\infty)} v\right\|_{L_{2,+}^{(0)}}^{2} \leq \frac{1}{2}\left\|b B_{0} \phi\right\|_{L_{2}\left(X_{\infty}\right)}^{2}
$$

Proof. Let $\phi_{j}$ be the solution of (3.1) as $H=H_{j}$. Then

$$
\|v\|_{L_{2,-}^{(0)}}^{2}=\left\|\mu \frac{\partial \phi_{j}}{\partial z}\right\|^{2}+\left\|\phi_{j}\right\|^{2}+\left\|S^{(j)} v\right\|_{L_{2,+}}^{2}-\left\|b B_{0} \phi_{j}\right\|^{2} \geq\left\|\phi_{j}^{0}\right\|_{H_{2}^{1}\left(X_{j}\right)}^{2}+\left\|S^{j} v\right\|_{L_{2,+}}^{2}-\left\|b B_{0} \phi_{j}\right\|_{L_{2}\left(X_{j}\right)}^{2},
$$

where $\phi_{j}^{0}$ is the solution of

$$
-\mu^{2} \frac{\partial^{2} \phi_{j}^{0}}{\partial z^{2}}+\phi_{i}^{0}=0 \text { in } X_{j}, \phi_{j}^{0}=v \text { on } \Gamma_{-}^{(j)}, \phi_{j}^{0}=S^{(j)} v \text { on } \Gamma_{+}^{(j)}
$$

Since

$$
\lim _{j \rightarrow \infty}\left\|\phi_{j}^{0}\right\|_{H_{2}^{1}\left(X_{j}\right)}^{2}=\|v\|_{L_{2,-}^{(0)}}^{2}+\left\|S^{(\infty)} v\right\|_{L_{2,+}^{(0)},}^{2}, \quad \lim _{j \rightarrow \infty}\left\|S^{(j)} v\right\|_{L_{2,+}}^{2}=\left\|S^{(\infty)} v\right\|_{L_{2,+}^{(0)}}^{2}
$$

then

$$
\|v\|_{L_{2,-}^{(0)}}^{2} \geq\|v\|_{L_{2,-}^{(0)}}^{2}+2\left\|S^{(\infty)} v\right\|_{L_{2,+}^{(0)}}^{2}-\left\|b B_{0} \phi\right\|_{L_{2}\left(X_{\infty}\right)}^{2},
$$

where $\phi=G_{1} v=\lim _{j \rightarrow \infty} \phi_{j}$ and (3.17) holds.

3.5. Now let us consider the problems with nontrivial $\operatorname{ker}(S)$. We begin the consideration from the case when $H=\infty$ and $S=S^{(\infty)}$.

Introduce the following set:

$$
K=\left\{\phi \in H_{2}^{1}:\left(\phi=G_{1} v\right) \bigcap\left(b B_{0} \phi=0 \forall z \geq 0\right), \quad v \in L_{2,-}^{(0)}\right\}
$$

The functions $\phi_{l}^{(m)}=\left\{P_{l}^{(m)} e^{i m \varphi} e^{-\frac{z}{\mu}}, \mu>0 ; 0, \mu<0\right\}, m \geq N+1$ belong to $K$. So, $K$ is nontrivial. It is easily seen also that all functions from $K$ satisfy the following relations

$$
\begin{gathered}
b \int_{0}^{2 \pi} \int_{0}^{1} p\left(z, \mu_{0}\right) v\left(\varphi^{\prime}, \mu^{\prime}\right) e^{\frac{-z}{\mu^{\prime}}} d \varphi^{\prime} d \mu^{\prime}=0 \forall z \geq 0, \\
\phi(\varphi, \mu, z)=\left\{v(\varphi, \mu) e^{\frac{-z}{\mu}}, \mu>0 ; 0, \mu<0\right\} .
\end{gathered}
$$


If $b>0, w_{l}(z)>0$, then

$$
\int_{0}^{1} P_{l}^{(m)}\left(\mu^{\prime}\right) \int_{0}^{2 \pi} e^{i m \varphi^{\prime}} v\left(\varphi^{\prime}, \mu^{\prime}\right) e^{\frac{-z}{\mu^{\prime}}} d \mu^{\prime} d \varphi^{\prime}=0 \forall z \geq 0, l=0,1, \ldots, N, \quad m=0,1, \ldots, l
$$

for $\forall \phi \in K$. From (3.17) and the properties of $K$ we have the following statements.

Lemma 3.5. Let $\phi=G_{1} v$ be a solution of (3.1) as $H=\infty$ and $v \in L_{2,-}^{(0)}$. (1) If $\phi \in K$, then $v \in \operatorname{ker}\left(S^{(\infty)}\right)$. (2) If $N<\infty$ in $p\left(z, \mu_{0}\right)=\sum_{i=0}^{N} w_{l}(z) P_{l}\left(\mu_{0}\right)$ then $\operatorname{ker}\left(S^{(\infty)}\right) \neq\{0\}$ and $\operatorname{dim}\left(\operatorname{ker}\left(S^{(\infty)}\right)\right)=\infty$. (3) If $\operatorname{ker}\left(S^{(\infty)}\right)=$ $\{0\}$ then $\phi=G_{1} v \notin K \forall v \in L_{2,-}^{(0)}$.

Let us represent the solution of the following adjoint problem

$$
A^{*} q=0 \text { in } X, q=w \text { on } \Gamma_{+},
$$

where $X=X_{\infty}, w \in L_{2,+}^{(0)}$, as $q=q_{T}+q_{R}$, where $q_{T}$ and $q_{R}$ are defined by

$$
\begin{gathered}
L^{*} q_{T}=0 \text { in } X, q_{T}=w \text { on } \Gamma_{+}, \\
L^{*} q_{R}=b B_{0} q_{R}+b B_{0} q_{T} \text { in } X, q_{R}=0 \text { on } \Gamma_{+} .
\end{gathered}
$$

Consider the following equality for the solution $\phi_{R}$ of (3.15) and $q$ :

$$
\left(b B_{0} \phi_{T}, q\right)+\left(\phi_{R}, q\right)_{L_{2,-}}=\left(\phi_{R}, q\right)_{L_{2,+}}+\left(\phi_{R}, A^{*} q\right) .
$$

Since $\phi_{R}=0$ on $\Gamma_{-}, A^{*} q=0$ in $X, q=q_{T}+q_{R}, q_{R}=0$ on $\Gamma_{+}$, then we have

$$
\left(b B_{0} \phi_{T}, q_{T}+q_{R}\right)=\left(\phi_{R}, q_{T}\right)_{L_{2,+}} .
$$

Assume that $p\left(z, \mu_{0}\right)=p\left(z,-\mu_{0}\right)$ and $v \in \operatorname{ker}\left(S^{(\infty)}\right) \neq\{0\}$, then $\phi_{R}=S^{(\infty)} v=0$ on $\Gamma_{+}$and

$$
\left(b B_{0} \phi_{T}, q_{T}\right)+\left(b B_{0} \phi_{T},\left(L^{*}-b B_{0}\right)^{-1} b B_{0} q_{T}\right)=0 .
$$

Let us set $w=U v$ in (3.19). It is easy to see that in this case $q_{T}=U \phi_{T}$. Since $B_{0} U \phi_{T}=B_{0} \phi_{T}$, then from (3.23)

$$
\left(b B_{0} \phi_{T}, \phi_{T}\right)+\left(b B_{0} \phi_{T},\left(L^{*}-b B_{0}\right)^{-1} b B_{0} \phi_{T}\right)=0, \quad\left(b B_{0} \phi_{T}, \phi_{T}\right)=0, \quad b B_{0} \phi_{T}=0 .
$$

From (3.24) and (3.17) we conclude that the following lemma is valid.

Lemma 3.6. Suppose that $p\left(z, \mu_{0}\right)=p\left(z,-\mu_{0}\right)$ and $0<b(z) \leq b_{1}<1$; then $v \in \operatorname{ker}\left(S^{(\infty)}\right)$ if and only if $\phi=G_{1} v \in K$.

Consider a specific case of the operator $B_{0}$. Assume that

$$
p\left(z, \mu_{0}\right)=p_{N}\left(z, \mu_{0}\right)+\varepsilon\left(p_{e v}\left(\mu_{0}\right)-p_{o d}\left(\mu_{0}\right)\right)
$$

where

$$
p_{N}\left(z, \mu_{0}\right)=p_{N}\left(z,-\mu_{0}\right)=\sum_{l=0}^{N} w_{l}(z) P_{l}\left(\mu_{0}\right), \quad 0 \leq w_{l}(z)<w_{0}=1
$$


$0<\varepsilon=$ const $\ll 1$ is a positive small value,

$$
p_{e v}\left(\mu_{0}\right)=p_{e v}\left(-\mu_{0}\right)=\sum_{l=0}^{\infty} \alpha^{2 l} P_{2 l}\left(\mu_{0}\right), \quad p_{o d}\left(\mu_{0}\right)=-p_{o d}\left(-\mu_{0}\right)=\sum_{l=1}^{\infty} \alpha^{2 l-1} P_{2 l-1}\left(\mu_{0}\right)
$$

and $0<\alpha<1$ is a given constant. We set

$$
B_{0} \phi=\frac{1}{4 \pi} \int_{0}^{2 \pi} \int_{-1}^{1} p\left(z, \mu_{0}\right) \phi\left(\varphi^{\prime}, \mu^{\prime}, z\right) d \varphi^{\prime} d \mu^{\prime}
$$

where $p\left(z, \mu_{0}\right)$ is given by $(3.25)$. Let us write $B_{0} \phi$ in the following form

$$
B_{0} \phi=B_{e v} \phi-\varepsilon B_{o d} \phi
$$

where

$$
B_{e v} \phi=\frac{1}{4 \pi} \int_{0}^{2 \pi} \int_{-1}^{1}\left(p_{N}+\varepsilon p_{e v}\right) \phi d \varphi^{\prime} d \mu^{\prime}, \quad B_{o d} \phi=\frac{1}{4 \pi} \int_{0}^{2 \pi} \int_{-1}^{1} p_{o d} \phi d \varphi^{\prime} d \mu^{\prime} .
$$

Lemma 3.7. If $b>0$ and $p\left(z, \mu_{0}\right)$ is given by $(3.25)$, then $\operatorname{ker}\left(S^{(\infty)}\right)=\{0\}$.

Proof. Assume that $\operatorname{ker}\left(S^{(\infty)}\right) \neq\{0\},(v \not \equiv 0) \in \operatorname{ker}\left(S^{(\infty)}\right)$ and $w=U v$ in (3.19). From (3.22) we obtain the following equality:

$$
\left(b B_{0} \phi_{T}, U \phi_{T}\right)+\left(b B_{0} \phi_{T},\left(L^{*}-b B_{0}\right)^{-1} b B_{0} U \phi_{T}\right)=0 .
$$

Since $B_{0} U \phi_{T}=B_{e v} U \phi_{T}-\varepsilon B_{o d} U \phi_{T}=B_{e v} \phi_{T}+\varepsilon B_{o d} \phi_{T}$ then

$$
\left(b\left(B_{e v}+\varepsilon B_{o d}\right) \phi_{T}, \phi_{T}\right)+\left(b\left(B_{e v}-\varepsilon B_{o d}\right) \phi_{T},\left(L^{*}-b B_{0}\right)^{-1}\left(b\left(B_{e v}+\varepsilon B_{o d}\right) \phi_{T}\right)=0\right.
$$

or

$$
0=\left(b\left(B_{e v}+\varepsilon B_{o d}\right) \phi_{T}, \phi_{T}\right)+\left(\left(L^{*}-b B_{0}\right) \psi, \psi\right)-2 \varepsilon\left(b B_{o d} \phi_{T}, \psi\right)
$$

where $\psi=\left(L^{*}-b B_{0}\right)^{-1} b\left(B_{e v}+\varepsilon B_{o d}\right) \phi_{T}$. If $\varepsilon$ is sufficiently small, then from (3.27) we obtain the following relationships:

$$
\begin{aligned}
0= & \left(b\left(B_{e v} \phi_{T}, \phi_{T}\right)+\varepsilon\left(b B_{o d} \phi_{T}, \phi_{T}\right)+\frac{1}{2}\|\psi\|_{L_{2,-}}^{2}+\left(\left(I-b B_{0}\right) \psi, \psi\right)-\left(2 \varepsilon b B_{o d} \phi_{T}, \psi\right)\right. \\
\geq & \left(b B_{e v} \phi_{T}, \phi_{T}\right)+\varepsilon\left(b B_{o d} \phi_{T}, \phi_{T}\right)+\frac{1}{2}\|\psi\|_{L_{2,-}}^{2}+\left(1-b_{1}\right)\|\psi\|^{2}+\varepsilon\left(b B_{o d} \psi, \psi\right) \\
& -2 \varepsilon\left(b B_{o d} \phi_{T}, \phi_{T}\right)^{\frac{1}{2}}\left(b B_{o d} \psi, \psi\right)^{\frac{1}{2}} \\
\geq & \left(b B_{e v} \phi_{T}, \phi_{T}\right)+\varepsilon\left(b B_{o d} \phi_{T}, \phi_{T}\right)+\frac{1}{2}\|\psi\|_{L_{2,-}}^{2}+\left(1-b_{1}\right)\|\psi\|^{2}+\varepsilon\left(b B_{o d} \psi, \psi\right)-\varepsilon\left(b B_{o d} \phi_{T}, \phi_{T}\right) \\
& -\varepsilon\left(b B_{o d} \psi, \psi\right)=\left(b B_{e v} \phi_{T}, \phi_{T}\right)+\frac{1}{2}\|\psi\|_{L_{2,-}}^{2}+\left(1-b_{1}\right)\|\psi\|^{2} .
\end{aligned}
$$

Hence, $\psi=0, \phi_{T}=0$ and $v=0$. But, by assumption, $v \not \equiv 0$. This contradiction proves the statement of this lemma.

3.6. Let $H$ be finite and

$$
\begin{gathered}
\phi_{T}=\left\{v_{1}(\varphi, \mu) e^{-z / \mu}, \mu>0 ; 0, \mu<0\right\} \\
K=\left\{\phi \in H_{2}^{1}:\left(\phi=G_{1} v\right) \bigcap\left(b B_{0} \phi=0 \forall z \geq 0\right), v \in L_{2,-}^{(0)}\right\} .
\end{gathered}
$$


Lemma 3.8. If $p\left(z, \mu_{0}\right)=p\left(z,-\mu_{0}\right)$ and $B_{0}$ is nonnegative then $v \in \operatorname{ker}\left(S_{R}\right)$ iff $\phi=G_{1} v \in K$. If $p\left(z, \mu_{0}\right)$ is of the form (3.25) then $\operatorname{ker}\left(S_{R}\right)=\{0\}$.

Proof. Let $q$ be the solution of (3.18), where $w=U v, v \in \operatorname{ker}\left(S_{R}\right)$. Then again,

$$
\begin{gathered}
0=\left(b B_{0} \phi_{T}, q_{T}+q_{R}\right)=\left(b B_{0} \phi_{T}, \phi_{T}\right)+\left(b B_{0} \phi_{T},\left(L^{*}-b B_{0}\right)^{-1} b B_{0} \phi_{T}\right), \\
\left(b B_{0} \phi_{T}, \phi_{T}\right)=0, \quad b B_{0} \phi_{T}=0
\end{gathered}
$$

and $\phi_{R}=0, \phi=G_{1} v=\phi_{T} \in K$. If $\phi=G_{1} v \in K$, then $\phi=\phi_{T}, \phi_{R}=0$ and $\phi_{R}=S_{R} v=0$ on $\Gamma_{+}$. So, $v \in \operatorname{ker}\left(S_{R}\right)$ iff $\phi=G_{1} v \in K$.

Assume that $p\left(z, \mu_{0}\right)$ has form (3.25), then repeating the proof of Lemma 3.8, we obtain other statements of this lemma.

Lemma 3.9. If $p\left(z, \mu_{0}\right)=p\left(z,-\mu_{0}\right), \quad H<\infty$ and $B_{0}$ is nonnegative, then $\operatorname{ker}\left(S_{1}\right)=\{0\}$.

Proof. Assume that $\operatorname{ker}\left(S_{1}\right) \neq\{0\}$ and $(v \not \equiv 0) \in\left(L_{2,-}^{(0)} \bigcap \operatorname{ker}\left(S_{1}\right)\right)$. Let $q$ be the solution of $(3.18)$ for $w=U v$. Then

$$
\begin{gathered}
0=(A \phi, q)=-(v, q)_{L_{2,-}},(v, q)_{L_{2,-}}=\left(v, q_{T}\right)_{L_{2,-}}+\left(v, q_{R}\right)_{L_{2,-}}, \\
\left(b B_{0} q_{T}, \phi\right)=\left(A^{*} q_{R}, \phi\right)=\left(q_{R}, v\right)_{L_{2,-}}, \\
0=\left(v, q_{T}\right)_{L_{2,-}}+\left(b B_{0} q_{T}, \phi\right)=\left(v, q_{T}\right)_{L_{2,-}}+\left(b B_{0} q_{T}, \phi_{T}\right)+\left(b B_{0} q_{T},\left(L-b B_{0}\right)^{-1} b B_{0} \phi_{T}\right) .
\end{gathered}
$$

Since $q_{T}=U \phi_{T}, v=\left(v_{1}, v_{2}\right) \equiv\left(v_{1}, 0\right) \in L_{2,-}^{(0)}$ and $\left(v, q_{T}\right)_{L_{2,-}}=0$ then

$$
\begin{gathered}
\left(b B_{0} \phi_{T}, \phi_{T}\right)+\left(b B_{0} \phi_{T},\left(L-b B_{0}\right)^{-1} b B_{0} \phi_{T}\right)=0, \quad\left(b B_{0} \phi_{T}, \phi_{T}\right)=0, \quad b B_{0} \phi_{T}=0, \quad \phi_{R}=0, \\
\phi=\phi_{T},\left.\quad \phi\right|_{\Gamma_{+}}=\left.\phi_{(T)}\right|_{\Gamma_{+}}=S_{1} v=0, \quad v=0 .
\end{gathered}
$$

So, we conclude that $\operatorname{ker}\left(S_{1}\right)=\{0\}$.

Let us summarize some of the above statements in the following theorems.

Theorem 3.2. Suppose that $H=\infty, 0 \leq b(z) \leq b_{1}=$ const $<1$; then for reflection operator $S: L_{2,-}^{(0)} \rightarrow L_{2,+}^{(0)}$ the following assertions hold: (1) $S=P_{(+)} \sum_{i=1}^{\infty}\left(L^{-1} b B_{0}\right)^{i} l^{-1}$ and $S$ is compact. (2) $\|S\|^{2} \leq\left(1-\sqrt{1-b_{1}^{2}}\right) /(1+$ $\left.\sqrt{1-b_{1}^{2}}\right)$. (3) If $p\left(z, \mu_{0}\right)=p\left(z,-\mu_{0}\right), 0<b(z) \leq b_{1}<1$ and $B_{0}$ is nonnegative then $v \in \operatorname{ker}(S)$ iff $\phi \equiv G_{1} v \in K$. (4) If $p\left(z, \mu_{0}\right)$ has the form (3.25) and $0<b(z) \leq b_{1}<1$, then $\operatorname{ker}(S)=\{0\}$.

Theorem 3.3. Suppoze that $H<\infty, 0 \leq b(z) \leq b_{1}=$ const $<1$; then for operator $S: L_{2,-} \rightarrow L_{2,+}$ the following assertions hold: (1) $S=S_{R}+S_{T}$, where $S_{T}=P_{(+)} l^{-1}: L_{2,-} \rightarrow L_{2,+}$ is a bounded and invertable operator, $S_{R}=P_{(+)} \sum_{i=1}^{\infty}\left(L^{-1} b B_{0}\right)^{i} l^{-1}: L_{2,-} \rightarrow L_{2,+}$ is compact. (2) $\|S\|^{2} \leq(1-q) /(1+q)$, where $q$ is given by (3.9). (3) If $p\left(z, \mu_{0}\right)=p\left(z,-\mu_{0}\right)$ and $B_{0}$ is nonnegative, then $\operatorname{ker}\left(S_{1}\right)=\{0\}$ and $v \in \operatorname{ker}\left(S_{R} P_{1}\right)$ iff $\phi \equiv G_{1} v \in K$. (4) If $p\left(z, \mu_{0}\right)$ is given by (3.25), then $\operatorname{ker}\left(S_{R} P_{1}\right)=\{0\}$. 
3.7. Consider now a specific radiative transfer problem - the boundary value problem for the function $\phi(\mu, z)$ $=(1 / 2 \pi) \int_{0}^{2 \pi} \phi(\varphi, \mu, z) d \varphi$ assuming $p\left(z, \mu_{0}\right) \equiv 1$ :

$$
\begin{gathered}
A_{0} \phi \equiv \mu \frac{\partial \phi}{\partial z}+\phi(\mu, z)-\frac{b(z)}{2} \int_{-1}^{1} \phi\left(\mu^{\prime}, z\right) d \mu^{\prime}=f(\mu, z) \text { in } X \equiv(-1,1) \times(0, H), \\
\phi=v_{1}(\mu), z=0, \mu>0 ; \quad \phi=v_{2}(\mu), z=H, \mu<0 .
\end{gathered}
$$

In the sequel we use the following lemma.

Lemma 3.10 [28]. If $f(x) \in L_{2}(0, \infty), v(\mu) \in L_{2}(0,1)$ and

$$
\begin{aligned}
& \int_{0}^{\infty} e^{-\frac{x}{\mu}} f(x) \frac{d x}{\mu}=0, \forall \mu \in(0,1), \\
& \int_{0}^{1} e^{-\frac{x}{\mu}} v(\mu) d \mu=0, \forall x \in(0, \infty),
\end{aligned}
$$

then $f(x)=0, v(\mu)=0$.

To investigate the problem (3.28) and the following boundary-value problem

$$
A_{0} \phi=0 \text { in } X, \quad \phi=v \text { on } \Gamma_{-}
$$

we introduce all "notions" which have been used earlier (functional spaces $H_{2}^{1}, \ldots, L_{2,-}^{(0)}, \Gamma_{-}, \Gamma_{+}, \ldots, S, S_{R}, S_{T}$ ). We mean here only that all these "notions" are independent of $\varphi \in(0,2 \pi]$. We point out that

$$
B_{0} \phi=\frac{1}{2} \int_{-1}^{1} \phi(\mu, z) d \mu
$$

Let us consider the problem (3.31) as $H \rightarrow \infty$ and introduce the set $K_{0}$ :

$$
K_{0}=\left\{\phi \in H_{2}^{1}:\left(\phi=G_{1} v\right) \bigcap\left(b B_{0} \phi\right)=0, \forall z \geq 0, \forall v \in L_{2,-}^{(0)}\right\}
$$

It is easily seen that $K_{0}=\{0\}$. Indeed, suppose that $v \in L_{2,-}^{(0)}, \phi=G_{1} v, b B_{0} \phi=0$. Then $\phi$ should have the following form: $\phi(\mu, z)=\left\{v(\mu) e^{-z / \mu}, \mu>0 ; 0, \mu<0\right\}$. Using the restriction $b B_{0} \phi=0$, we obtain $b \int_{0}^{1} e^{-z / \mu} v(\mu) d \mu=0, z \geq 0$. According to Lemma 3.10 we conclude that $v(\mu)=0$ and $K_{0}=\{0\}$.

Suppose that $\operatorname{ker}\left(S^{(\infty)}\right) \neq\{0\}, v \not \equiv 0 \in \operatorname{ker}\left(S^{(\infty)}\right)$. Then

$$
0=S v=\phi(\mu, 0)=\int_{0}^{\infty} e^{-\frac{x}{\mid \mu}} b(z) B_{0} \phi \frac{d x}{|\mu|}, \mu<0
$$


where $\phi=G_{1} v$. Using Lemma 3.10, we have: i) $b(z) B_{0} \phi=0$; ii) $\phi=\left\{v e^{-z / \mu}, \mu<0 ; \mu>0\right\}$; iii) $B_{0} \phi=\int_{0}^{1} v e^{-z / \mu} d \mu=0 \forall z \geq 0$; iv) $v(\mu)=0$. The contradiction proves the statement: $\operatorname{ker}\left(S^{(\infty)}\right)=\{0\}$. So, the following theorem is valid.

Theorem 3.4. Suppose that $H \rightarrow \infty$ and $0<b \leq b_{1}<1$ in the problem (3.31). Then: (1) $S^{(\infty)}$ is compact. (2) $\left\|S^{(\infty)}\right\|^{2} \leq\left(1-\sqrt{1-b_{1}^{2}}\right) /\left(1+\sqrt{1-b_{1}^{2}}\right)$. (3) $\operatorname{ker}\left(S^{(\infty)}\right)=\operatorname{ker}\left(\left(S^{(\infty)}\right)^{*}\right)=\{0\}$. (4) There exists the inverse operator $\left(S^{(\infty)}\right)^{-1}$, which is not bounded on the range $R\left(S^{(\infty)}\right)$. (5) The range $R\left(S^{(\infty)}\right)$ is dense in $L_{2,+}^{(0)}$.

(The proof of this theorem follows from the above considerations and the theory of compact operators.)

\section{Solvability OF INVERSE PROBlEMS}

4.1. With the use of the above operators problem (2.11) can be written in the following form:

Problem 1. Given $\phi^{(0)} \in H_{2}^{1}, \phi_{\mathrm{obs}} \in L_{2,+}$, find $\phi \in H_{2}^{1}, q \in H_{2}^{1}, v \in L_{2,-}$ such that

$$
\begin{gathered}
A \phi=0 \text { in } X, \phi=v \text { on } \Gamma_{-}, \\
A^{*} q=0 \text { in } X, q=\phi-\left(\phi_{\text {obs }}-\phi^{(0)}\right) \text { on } \Gamma_{+}, \\
\alpha v+S^{*} P_{(+)} q=0 \text { on } \Gamma_{-}
\end{gathered}
$$

or as the equation for $v$ : given $\phi_{\mathrm{obs}}$, find $v \in L_{2,-}$ such that

$$
\alpha v+S^{*} S v=S^{*} P_{(+)}\left(\phi_{\mathrm{obs}}-\phi^{(0)}\right) \text { on } \Gamma_{-}
$$

or as a weak form of (4.2): find $v \in L_{2,-}$ such that

$$
\alpha(v, w)_{L_{2,-}}+(S v, S w)_{L_{2,+}}=\left(P_{(+)}\left(\phi_{\mathrm{obs}}-\phi^{(0)}\right), S w\right)_{L_{2,+}} \forall w \in L_{2,-} .
$$

If $H \rightarrow \infty, P_{(+)}\left(\phi_{\mathrm{obs}}-\phi^{(0)}\right) \in P_{1} L_{2,+}=L_{2,+}^{(0)}, v \in L_{2,-}^{(0)}$ in $(2.11)$, then we have the second inverse problem.

Problem 2. Given $P_{(+)}\left(\phi_{\mathrm{obs}}-\phi^{(0)}\right) \in L_{2,+}^{(0)}$ find $v \in L_{2,-}^{(0)}$ such that

$$
\alpha(v, w)_{L_{2,-}^{(0)}}+\left(S^{(\infty)} v, S^{(\infty)} w\right)_{L_{2,+}^{(0)}}=\left(P_{(+)}\left(\phi_{\mathrm{obs}}-\phi^{(0)}\right), S^{(\infty)} w\right)_{L_{2,+}^{(0)}} \forall w \in L_{2,-}^{(0)}
$$

or forms $(4.1,4.2)$ with $S, S^{*}$ replaced by $S^{(\infty)},\left(S^{(\infty)}\right)^{*}$ hold.

4.2. Using the properties of the above eflection operators, we formulate some statements on the solvability of Problems 1, 2.

Throughout this section we assume $\operatorname{ker}(S)=\operatorname{ker}\left(S^{*}\right)=\{0\}$ (see, Ths. 3.2-3.4) and we consider only this case of Problems 1, 2.

Some solvability results will be proved in the presence of additional restrictions and the following remark may be useful. It is easy to see that the following assumptions are equivalent: (i) There exists a unique solution $\phi_{0} \in H_{2}^{1}, v_{0} \in L_{2,-}$ of (4.1) as $\alpha=0$; (ii) $S^{-1} P_{(+)}\left(\phi_{\mathrm{obs}}-\phi^{(0)}\right) \in L_{2,-}$; (iii) there exists a function $\phi_{1} \in H_{2}^{1}$ such that $A \phi_{1}=0$ in $X$ and $\phi_{1}=P_{(+)}\left(\phi_{\text {obs }}-\phi^{(0)}\right)$ on $\Gamma_{+}$.

Now let us formulate some conclusions from statements of the previous sections. The domain $D(S)$ of $S$ is the whole space $L_{2,-}$, the range $R(S)$ is a subset of $L_{2,+}, D\left(S^{*}\right)=L_{2,+}, R\left(S^{*}\right) \subset L_{2,-}$ and the operators $S, S^{*}$ are closed. Since $L_{2,-}, L_{2,+}$ are Hilbert spaces, $S, S^{*}$ are closed and $\operatorname{ker}(S)=\operatorname{ker}\left(S^{*}\right)=\{0\}$ 
then $\overline{R(S)}=L_{2,+}, \overline{R\left(S^{*}\right)}=L_{2,-}$. However, $S^{-1},\left(S^{*}\right)^{-1}$ are unbounded for any $H \leq \infty$. Therefore $R(S)$ $\neq L_{2,+}, R\left(S^{*}\right) \neq L_{2,-}$ and $S, S^{*}$ are not "normal solvable" and not Fredholm operators. Hence, the equations $S v=g, S^{*} q=g_{1}$ can be "dense solvable" only (if additional restrictions to $g \in L_{2,+}, g_{1} \in L_{2,-}$ are not imposed) and Problems 1, 2 are ill-posed.

Let $H_{S}$ be the completion of $L_{2,-}$ with respect to norm $\|v\|_{H_{S}} \equiv\|S v\|_{L_{2,+}}$. Since $\overline{R(S)}=L_{2,+}$ then for any $v \in H_{S}$ there exists a function $\phi \in L_{2,+}$ such that $S v=\phi$ and $\|v\|_{H_{S}}=\|\phi\|_{L_{2,+}}$; if $v \in L_{2,-}$ then $\phi \in R(S)$. So, the spaces $H_{S}, L_{2,+}$ are isometric. Above remarks will be used below in proving solvability results.

Let us consider the equation (4.2). Using properties of $S$ we conclude that the following statement holds.

Theorem 4.1. The following assertions hold:

(1) If $\alpha>0$ then the inverse problem $(4.2)((4.1),(2.8))$ has a unique solution and the estimates

$$
\begin{gathered}
\|\phi\|_{H_{2}^{1}}+\|v\|_{L_{2,-}} \leq C\left(\|f\|+\left\|\phi_{\mathrm{obs}}\right\|_{L_{2,+}}\right) \\
(1+\alpha)\|\phi\|_{L_{2,+}}^{2}+\alpha\|v\|_{L_{2,-}}^{2}+2 \alpha\left(1-b_{1}\right)\|\phi\|^{2} \leq\left\|P_{(+)}\left(\phi_{\mathrm{obs}}-\phi^{(0)}\right)\right\|^{2}
\end{gathered}
$$

are valid, $C=C(\alpha)=$ const $<\infty$.

(2) If $\alpha=0$ and $\phi_{\mathrm{obs}, 0} \equiv P_{(+)}\left(\phi_{\mathrm{obs}}-\phi^{(0)}\right)$ is a function from $L_{2,+}$ then $(4.2)$ has a unique weak solution $v_{0}=\left(S^{*} S\right)^{-1} S^{*} \phi_{\mathrm{obs}, 0}$ and the estimate

$$
\left\|v_{0}\right\|_{H_{S}} \leq\left\|\phi_{\mathrm{obs}, 0}\right\|_{L_{2,+}}
$$

is valid. The function $v_{0}$ is a weak solution of the equation $S v=\phi_{\mathrm{obs}, 0}$ also, i.e. the relation

$$
(S v, w)_{L_{2,+}}=\left(\phi_{\mathrm{obs}, 0}, w\right)_{L_{2,+}} \quad \forall w \in L_{2,+}
$$

is satisfied. If $\phi_{\mathrm{obs}, 0} \in R(S)$ then $v_{0}=S^{-1} \phi_{\mathrm{obs}, 0}$ is the solution of "an exact controllability problem"; if $S^{-1} \phi_{\mathrm{obs}, 0} \in L_{2,-}$ then $v_{0} \in L_{2,-}$ also.

(3) There exists a sufficiently small positive $\alpha$ such that the solution $\phi \equiv \phi(v)$ of (4.1) satisfies

$$
\left\|\phi-\phi_{\mathrm{obs}, 0}\right\|_{L_{2,+}} \leq \epsilon
$$

for given $\epsilon>0$, i.e. (4.1) is the "approximate cotrollability problem" and it has a solution $\forall \phi_{\mathrm{obs}, 0} \in L_{2,+}$.

(4) If $\phi, v$ is a solution of (4.1) as $\alpha>0$ while $\phi_{0}, v_{0}$ is a solution of this problem as $\alpha=0$ then

$$
\left\|S^{*}\left(\phi-\phi_{0}\right)\right\|_{H_{S}}=\left\|S^{*} S\left(v-v_{0}\right)\right\|_{H_{S}} \leq \alpha\left\|\phi_{\mathrm{obs}, 0}\right\|_{L_{2,+}}
$$

for $\phi_{\mathrm{obs}, 0} \in L_{2,+}$ and

$$
\left\|\phi-\phi_{0}\right\|_{L_{2,+}}=\left\|v-v_{0}\right\|_{H_{S}} \leq(\alpha / 2)^{1 / 2}\left\|S^{-1} \phi_{\mathrm{obs}, 0}\right\|_{L_{2,-}}
$$

for $S^{-1} \phi_{\mathrm{obs}, 0} \in L_{2,-}$.

Proof. 1. Since

$$
\left(\alpha v+S^{*} S v, v\right)_{L_{2,-}}=\alpha\|v\|_{L_{2,-}}^{2}+\|S v\|_{L_{2,+}}^{2},
$$

then the problem (4.2) is correctly solvable and

$$
\|v\|_{L_{2,-}}^{2} \leq \frac{1}{\alpha}\left\|P_{(+)}\left(\phi_{\mathrm{obs}}-\phi^{(0)}\right)\right\|_{L_{2,+}}\|S v\|_{L_{2,+}} \leq \frac{1}{\alpha}\left\|\phi_{\mathrm{obs}}-\phi^{(0)}\right\|_{L_{2,+}}\|S\|\|v\|_{L_{2,-}} \leq \frac{\gamma_{1}^{1 / 2}}{\alpha}\left\|\phi_{\mathrm{obs}}-\phi^{(0)}\right\|_{L_{2,+}}\|v\|_{L_{2,-}}
$$


So,

$$
\|v\|_{L_{2,-}} \leq \frac{\gamma_{1}^{1 / 2}}{\alpha}\left\|\phi_{\mathrm{obs}}-\phi^{(0)}\right\|_{L_{2,+}}
$$

and the estimate $(4.5)$ is valid.

Let us consider now a weak statement of the boundary value problem for $\phi$ :

$$
\left(\phi,-\mu \frac{\partial w}{\partial z}+w-b B_{0} w\right)+(\phi, w)_{L_{2,+}}+\frac{1}{\alpha}\left(S^{*} P_{(+)} \phi, w\right)_{L_{2,-}}=\frac{1}{\alpha}\left(S^{*} P_{(+)}\left(\phi_{\mathrm{obs}}-\phi^{(0)}, w\right)\right)_{L_{2,-}},
$$

where $\forall w \in H_{2}^{1}$ and let us use the boundary condition for $\phi$ in the following form:

$$
\phi+\frac{1}{\alpha} S^{*} P_{(+)}\left(\phi-\left(\phi_{\mathrm{obs}}-\phi^{(0)}\right)\right)=0 \text { on } \Gamma_{-} .
$$

Setting $w=\phi$, we have:

$$
\left.\frac{1}{2}\|\phi\|_{L_{2,+}}^{2}+\frac{1}{2}\|\phi\|_{L_{2,-}}^{2}+(T \phi, \phi)+\frac{1}{\alpha}\left(S^{*} P_{(+)} \phi, \phi\right)_{L_{2,-}}=\frac{1}{\alpha}\left(S^{*} P_{(+)}\left(\phi_{\mathrm{obs}}-\phi^{(0)}\right), \phi\right)\right)_{L_{2,-}} .
$$

Since

$$
\begin{gathered}
\left(S^{*} P_{(+)} \phi, \phi\right)_{L_{2,-}}=\|\phi\|_{L_{2,+}}^{2}, \quad\left|\left(S^{*} P_{(+)}\left(\phi_{\mathrm{obs}}-\phi\right), \phi\right)_{L_{2,-}}\right|=\left|\left(P_{(+)}\left(\phi_{\mathrm{obs}}-\phi^{(0)}\right), \phi\right)_{L_{2,+}}\right| \\
\leq\left\|\phi_{\mathrm{obs}}-\phi^{(0)}\right\|_{L_{2,+}}\|\phi\|_{L_{2,+}} \leq \frac{1}{2}\left\|\phi_{\mathrm{obs}}-\phi^{(0)}\right\|_{L_{2,+}}^{2}+\frac{1}{2}\|\phi\|_{L_{2,+}}^{2}, \\
(T \phi, \phi) \geq\left(1-b_{1}\right)\|\phi\|^{2},
\end{gathered}
$$

then

$$
(1+\alpha)\|\phi\|_{L_{2,+}}^{2}+\alpha\|\phi\|_{L_{2,-}}^{2}+2 \alpha\left(1-b_{1}\right)\|\phi\|^{2} \leq\left\|\phi_{\mathrm{obs}}-\phi^{(0)}\right\|_{L_{2,-}}^{2},
$$

i.e. we have obtained the second estimate from (4.5).

2. If $\alpha=0$ then (4.2) is equivalent to the following equation

$$
S^{*} S v_{0}=S^{*} \phi_{\mathrm{obs}, 0} .
$$

By usual considerations of the variational problem theory, the existence of a unique weak solution $v_{0}=\left(S^{*} S\right)^{-1} S^{*} \phi_{\mathrm{obs}, 0}$ of (4.10) and the estimate (4.6) are proved. Since $R(S) \subset D\left(S^{*}\right)=L_{2,+}$ and $\operatorname{ker}\left(S^{*}\right)=0$ then (4.10) is equivalent to the equation $S v_{0}=\phi_{\mathrm{obs}, 0}$, i.e. $v_{0}$ is a weak solution of this equation also. If $\phi_{\mathrm{obs}, 0} \in R(S)$ then $v_{0}=S^{-1} \phi_{\mathrm{obs}, 0}$ and $v_{0} \in L_{2,-}$ for $S^{-1} \phi_{\mathrm{obs}, 0} \in L_{2,-}$.

3. The proof of this assertion is similar to proof from [35] (p. 285). Let $\phi_{\mathrm{obs}, 0}$ be an arbitrary function from $L_{2,+}$. Since $\overline{R(S)}=L_{2,+}$ then there exists $w \in L_{2,-}$ such that $\left\|\phi(w)-\phi_{\mathrm{obs}, 0}\right\|_{L_{2,+}} \leq \epsilon_{1}$ for given $\epsilon_{1}>0$, where $\phi \equiv \phi(w)$ is the solution of

$$
A \phi=0 \quad \text { in } \quad X, \quad \phi=w \text { on } \Gamma_{-} .
$$

If $\phi=\phi(v), v$ is a solution of (4.1) for $\alpha>0$ then

$$
J(\phi(v), v) \leq J(\phi(w), w) \leq \alpha\|w\|_{L_{2,-}}^{2}+\epsilon_{1}^{2} .
$$


Let us fix $\epsilon_{1}, w$ and choose a sufficiently small $\alpha$ such that $\alpha\|w\|_{L_{2,-}}^{2} \leq \epsilon_{1}^{2}$. Then

$$
\left\|\phi(v)-\phi_{\mathrm{obs}, 0}\right\|_{L_{2,+}}^{2} \leq J(\phi(v), v) \leq 2 \epsilon_{1}^{2} \equiv \epsilon^{2} .
$$

The last relation implies (4.7).

4. It is easy to see that

$$
S S^{*} S\left(v-v_{0}\right)=-\alpha\left(\alpha\left(S S^{*}\right)^{-1}+I\right) \phi_{\mathrm{obs}, 0}
$$

If $\phi_{\mathrm{obs}, 0} \in L_{2,+}$ then

$$
\left\|S S^{*} S\left(v-v_{0}\right)\right\|_{L_{2,+}}=\left\|S^{*} S\left(v-v_{0}\right)\right\|_{H_{S}} \leq \alpha\left\|\phi_{\mathrm{obs}, 0}\right\|_{L_{2,+}} .
$$

Let us write down now the following equalities

$$
\begin{gathered}
v=\left(\alpha I+S^{*} S\right)^{-1} S^{*} \phi_{\mathrm{obs}, 0}=\left(\alpha I+S^{*} S\right)^{-1} S^{*} S v_{0}, \\
\alpha(v, v)_{L_{2,-}}+\left\|v-v_{0}\right\|_{H_{S}}^{2}=\alpha\left(v, v_{0}\right)_{L_{2,-}} .
\end{gathered}
$$

If $S^{-1} \phi_{\mathrm{obs}, 0} \in L_{2,-}$ then $\|v\|_{L_{2,-}} \leq\left\|v_{0}\right\|_{L_{2,-}}$ and (4.9) is a simple consequence of (4.11).

4.3. In Problem 2 the operators $S=S^{(\infty)}, S^{*}=\left(S^{(\infty)}\right)^{*}$ are compact and $\left(\alpha I+S^{*} S\right)$ is a "normal solvable" and Fredholm operator. Hence, some special theoretical approaches can be used to develope Problem 2 (for example, singular spectral expansions of operators, etc.). But the principal solvability results remain the same as in Problem 1.

Theorem 4.2. The following statements are valid:

(1) Problem 1 for $\alpha>0$ is equivalent to the second-type operator equation for $v$ with Fredholm operator and to the first-type equation with compact operator at $\alpha=0$.

(2) If $\alpha>0$ then Problem 2 has a unique solution for $\phi_{\mathrm{obs}, 0} \equiv P_{(+)}\left(\phi_{\mathrm{obs}}-\phi^{(0)}\right) \in L_{2,+}^{(0)}$ and the estimate (4.5) is valid. If $\alpha=0, P_{(+)}\left(\phi_{\mathrm{obs}}-\phi^{(0)}\right) \in R\left(S^{(\infty)}\right)$ then there exists a unique solution of Problem 2:

$$
v=\left(S^{(\infty)}\right)^{-1}\left(P_{(+)}\left(\phi_{\mathrm{obs}}-\phi^{(0)}\right)\right)
$$

i.e. "the problem of exact controllability" has a unique solution.

(3) If $\phi_{\mathrm{obs}, 0}$ is an arbitrary function from $L_{2,+}^{(0)}$ then there exists a sufficiently small $\alpha>0$ such that the solution $\phi=\phi(v)$ of $(4.2)((2.11),(4.1))$ for $\alpha>0$ satisfies

$$
\left\|\phi-\phi_{\mathrm{obs}, 0}\right\|_{L_{2,+}^{(0)}} \leq \epsilon
$$

for given $\epsilon>0$.

(4) The estimates

$$
\left\|\left(S^{(\infty)}\right)^{*}\left(\phi-\phi_{0}\right)\right\|_{H_{S}} \leq \alpha\left\|\phi_{\mathrm{obs}, 0}\right\|_{L_{2,+}^{(0)}}
$$

where $\phi_{\mathrm{obs}, 0} \in L_{2,+}^{(0)}$, and

$$
\left\|\phi-\phi_{0}\right\|_{L_{2,+}^{(0)}}=\left\|v-v_{0}\right\|_{H_{S}} \leq(\alpha / 2)^{1 / 2}\left\|\left(S^{(\infty)}\right)^{-1} \phi_{\mathrm{obs}, 0}\right\|_{L_{2,-}^{(0)}},
$$

where $\left(S^{(\infty)}\right)^{-1} \phi_{\mathrm{obs}, 0} \in L_{2,-}^{(0)}$, are valid. 
(The proof of Th. 4.2 repeats in the whole the proof of the previous theorem.)

Remark. Consider the system of equations given by (4.1). Assume that $\alpha=0, P_{(+)}\left(\phi_{\mathrm{obs}}-\phi^{(0)}\right) \in R\left(S^{(\infty)}\right)$ and there exist $S^{-1},\left(S^{*}\right)^{-1}$. Then from $(4.1)$ we obtain: $P_{(+)} q=0, \phi=\left(\phi_{\mathrm{obs}}-\phi^{(0)}\right)$ on $\Gamma_{+}, v=S^{-1} P_{(+)}\left(\phi_{\mathrm{obs}}-\right.$ $\left.\phi^{(0)}\right)$ on $\Gamma_{-}$and

$$
A \phi=0 \text { in } X, \phi=S^{-1} P_{(+)}\left(\phi_{\text {obs }}-\phi^{(0)}\right) \text { on } \Gamma_{-}, \phi=\phi_{\text {obs }}-\phi^{(0)} \text { on } \Gamma_{+},
$$

i.e. "the exact controllability problem" has a solution. Assume that some function $\phi \in H_{2}^{1}$ satisfies (4.14). Then from (4.14) and (4.1):

$$
v=S^{-1} P_{(+)}\left(\phi_{\mathrm{obs}}-\phi^{(0)}\right) \text { on } \Gamma_{-}, q=0 \text { in } X, \alpha v=0 \text { on } \Gamma_{-} .
$$

If $v \not \equiv 0$, then $\alpha$ should be equal to zero. So, we conclude: the exact controllability in (4.1) is admitted iff $\alpha=0$ and $P_{(+)}\left(\phi_{\text {obs }}-\phi^{(0)}\right) \in R\left(S^{(\infty)}\right)$.

\section{ON NUMERICAL SOLUTION OF INVERSE PROBLEMS}

Statements of the previous section allow to conclude that an approximate solution of Problem 1 (2) calculated for small $\alpha>0$, can be considered as an approximate solution of this problem as $\alpha=0$ also. To develope appropriate approximate solutions of Problems 1, 2 for $\alpha>0$ various iterative algorithms can be used [28-31]. Below we consider an example of such procedures.

To construct an approximate solution of (4.1) we can apply the iterative algorithm given by:

$$
\begin{gathered}
A \phi_{n}=0 \text { in } X, \quad \phi_{n}=v_{n} \text { on } \Gamma_{-}, \\
A^{*} q_{n}=0 \text { in } X, \quad q_{n}=\phi_{n}-\left(\phi_{\text {obs }}-\phi^{(0)}\right) \text { on } \Gamma_{+}, \\
v_{n+1}=v_{n}-\tau\left(\alpha v_{n}+q_{n}\right) \text { on } \Gamma_{-}, \quad n=0,1, \ldots,
\end{gathered}
$$

where $v_{0} \in L_{2,-}, \tau=2 /\left(2 \alpha+\gamma_{1}\right)$ and $\gamma_{1}$ is given by (3.8).

In view of the reflection operator properties and the iterative processes theory results,

Lemma 5.1 [28]. If $\alpha>0$ and $\tau=2 /\left(2 \alpha+\gamma_{1}\right)$, then the algorithm (5.1) converges and the estimate

$$
\left\|\phi-\phi_{n}\right\|_{H_{2}^{1}}+\left\|q-q_{n}\right\|_{H_{2}^{1}}+\left\|v-v_{n}\right\|_{L_{2,-}} \leq C\left(\frac{\gamma_{1}}{2 \alpha+\gamma_{1}}\right)^{n}\left\|v-v_{0}\right\|_{L_{2,-}}
$$

is true, where $C=$ const $>0$.

To solve the subproblems from (5.1) we can use known numerical methods $[2,5,6,9,10]$. Since the errors of these methods are well-known then we can investigate the convergence property of (5.1) taking into account also numerical errors. But we do not discuss this issue in the paper.

\section{CONCLuSion}

Let us summarize some results formulated in this paper. We considered the methodology of analysis and numerical solution of some inverse radiative problems. This methodology is based on the optimal control theory 
approaches, solvability results for first- or second-type operator equations and on modern iterative processes. We hope that it will be applied to other inverse problems for transport equations.

We saw that the crucial issue of solvability and numerical solution of such problems is connected with properties of reflection operetors $S$, which are investigated in the "direct" boundary-value problems for transport equations. If $S$ consists of bounded and compact parts then the theory of second-type equations is a basis for consideration of an inverse problem; if $S$ is compact then results for the first-type operator equation is such basis.

The exact controllability problems considered above are rather specific. If $S$ is compact, $\operatorname{ker}(S)=0$ and $P_{(+)}\left(\phi_{\text {obs }}-\phi^{(0)}\right) \in R(S)$ then each of such problems has a unique solution. One of the practical implications of this statement is the following: since $S^{-1}$ is unbounded then the considered inverse problem is ill-posed and various regularization procedures are useful in numerical procedures. If $P_{(+)}\left(\phi_{\text {obs }}-\phi^{(0)}\right)$ is a function from $L_{2,+}$ then (1.3) has no solution, but it can possess a generalized solution, and (1.3) should be replaced by a weak statement (like $(2.8)$ ). The presence of estimates $(4.7-4.9,4.13)$ is important in numerical practice and it means that approximate solutions of inverse problems can be obtained for $P_{(+)}\left(\phi_{\text {obs }}-\phi^{(0)}\right) \in L_{2,+}$.

Let us conclude this paper with remarks that can useful also in practical calculations.

If $\alpha>0$, then equation (4.2) has a "good" operator $\left(\alpha I+S^{*} S\right)$. Using the iterative processes theory, it is possible to write a lot of iterative algorithms to solve (4.1). We can also optimize the convergence rate of these algorithms using properties of $S, S^{*}$ presented early [29-31].

To solve (4.1) as $H \rightarrow \infty, v \in L_{2,-}^{(0)},\left(\phi_{\text {obs }}-\phi^{(0)}\right) \in L_{2,+}^{(0)}$ the process (5.1) (or some others algorithms) can be applied also. If $P_{(+)}\left(\phi_{\mathrm{obs}}-\phi^{(0)}\right) \in R\left(S^{(\infty)}\right)$, then there is a possibility to set $\alpha=0$. However, numerical calculations can be unstable in this case.

Assume that $p \equiv p_{N}\left(z, \mu_{0}\right)=p_{N}\left(z,-\mu_{0}\right)$ is considered in the original statement of boundary value problems. To have the property " $\operatorname{ker}(S)=0$ " we can replace this $p\left(z, \mu_{0}\right)$ by the perturbed one of form (3.25). This perturbation of the problems can be considered also as a regularization of inverse problems considered above.

The present investigation has shown the potential of optimal control approaches for investigation and numerical solution of inverse radiative transfer problems and has suggested many directions for further work.

\section{REFERENCES}

[1] V.A. Ambartsumyan, Scattering and absorption of light in planetary atmospheres. Uchen. Zap. TsAGI 82 (1941), in Russian.

[2] S. Chandrasekhar, Radiative Transfer. New York (1960).

[3] J.-L. Lions, Contrôle optimal des systèmes gouvernés par des équations aux dérivées partielles. Dunod, Paris (1968).

[4] V.I. Lebedev and V.I. Agoshkov, The Poincaré-Steklov Operators and their Applications in Analysis. Dept. of Numerical Math. of the USSR Academy of Sciences, Moscow (1983), in Russian.

[5] V.I. Agoshkov, Generalized solutions of transport equations and their smoothness properties. Nauka, Moscow (1988), in Russian.

[6] V.I. Agoshkov, Reflection operators and domain decomposition methods in transport theory problems. Sov. J. Numer. Anal. Math. Modelling 2 (1987) 325-347.

[7] V.I. Agoshkov, On the existence of traces of functions in spaces used in transport theory problems. Dokl. Akad. Nauk SSSR 288 (1986) 265-269, in Russian.

[8] V.S. Vladimirov, Mathematical problems of monenergetic particle transport theory. Trudy Mat. Inst. Steklov 61 (1961), in Russian.

[9] G.I. Marchuk, Design of Nuclear Reactors. Atomizdat, Moscow (1961), in Russian.

[10] V.V. Sobolev, Light Scattering in Planetary Atmospheres. Pergamon Press, Oxford, U.K. (1973).

[11] G.I. Marchuk and V.I. Agoshkov, Reflection Operators and Contemporary Applications to Radiative Transfer. Appl. Math. Comput. 80 (1995) 1-19.

[12] V.I. Agoshkov, Domain decomposition methods in problems of hydrodynamics. I. Problem plain circulation in ocean. Moscow: Department of Numerical Mathematics, Preprint No. 96 (1985) 12, in Russian.

[13] V.I. Agoshkov, Domain decomposition methods and perturbation methods for solving some time dependent problems of fluid dynamics, in Proc. of First International Interdisciplinary Conference. Olympia -91 (1991).

[14] V.I. Agoshkov, Control theory approaches in: data assimilation processes, inverse problems, and hydrodynamics. Computer Mathematics and its Applications, HMS/CMA 1 (1994) 21-39.

[15] Ill-posed problems in natural Sciences, edited by A.N. Tikhonov. Moscow, Russia - VSP, Netherlands (1992). 
[16] A.L. Ivankov, Inverse problems for the nonstationary kinetic transport equation. In [15].

[17] A.I. Prilepko, D.G. Orlovskii and I.A. Vasin, Inverse problems in mathematical physics. In [15].

[18] Yu.E. Anikonov, New methods and results in multidimensional inverse problems for kinetic equations. In [15].

[19] E.C. Titchmarsh, Introduction to the Theory of Fourier Integral. New York (1937).

[20] C. Bardos, Mathematical approach for the inverse problem in radiative media (1986), not published.

[21] K.M. Case, Inverse problem in transport theory. Phys. Fluids 16 (1973) 16-7-1611.

[22] L.P. Niznik and V.G. Tarasov, Reverse scattering problem for a transport equation with respect to directions. Preprint, Institute of Mathematics, Academy Sciences of the Ukrainian SSR (1980).

[23] K.K. Hunt and N.J. McCormick, Numerical test of an inverse method for estimating single-scattering parameters from pulsed multiple-scattering experiments. J. Opt. Soc. Amer. A. 2 (1985).

[24] N.J. McCormick, Recent Development in inverse scattering transport method. Trans. Theory Statist. Phys. 13 (1984) 15-28.

[25] C. Bardos, R. Santos and R. Sentis, Diffusion approximation and the computation of critical size. Trans. Amer. Math. Soc. 284 (1986) 617-649.

[26] C. Bardos, R. Caflish and B. Nicolaenko, Different aspect of the Milne problem. Trans. Theory Statist. Phys. 16 (1987) $561-585$.

[27] V.P. Shutyaev, Integral reflection operators and solvability of inverse transport problem, in Integral equations in applied modelling. Kiev: Inst. of Electrodynamics, Academy of Sciences of Ukraine, Vol. 2 (1986) 243-244, in Russian.

[28] V.I. Agoshkov and C. Bardos, Inverse radiative problems: The problem on boundary function. CMLA, ENS de Cachan, Preprint No. 9801 (1998).

[29] V.I. Agoshkov and C. Bardos, Inverse radiative problems: The problem on the right-hand-side function. CMLA, ENS de Cachan, Preprint No. 9802 (1998).

[30] V.I. Agoshkov and C. Bardos, Optimal control approach in 3D-inverse radiative problem on boundary function (to appear).

[31] V.I. Agoshkov, C. Bardos, E.I. Parmuzin and V.P. Shutyaev, Numerical analysis of iterative algorithms for an inverse boundary transport problem (to appear).

[32] S.I. Kabanikhin and A.L. Karchevsky, Optimization methods of solving inverse problems of geoelectrics. In [15].

[33] F. Coron, F. Golse and C. Sulem, A Classification of Well-Posed Kinetic Layer Problems. Comm. Pure Appl. Math. 41 (1988) 409-435.

[34] R. Dautray and J.L. Lions, Analyse mathématique et calcul numérique pour les sciences et les techniques, CEA. Masson, Tome 9.

[35] R. Glowinski and J.-L. Lions, Exact and approximate controllability for distributed parameter systems. Acta Numer. (1994) 269-378. 